\title{
A common policy framework for evidence generation on promising health technologies
}

\author{
Cédric Carbonneil, Fabienne Quentin, Sun Hae Lee-Robin \\ French National Authority for Health \\ for the European network for Health Technology Assessment
(EUnetHTA)
}

Background: Generation of additional evidence may be necessary to access new promising technologies (marketing approval or coverage). Access with evidence generation $(A E G)$ is a more recent concept with regard to coverage than to marketing approval.

Objectives: One aim of Work Package 7 (WP7) Strand A of the European network for Health Technology Assessment (EUnetHTA) was to provide an overview of national AEG mechanisms associated with marketing approvals and funding or coverage decisions.

Methods: A systematic literature review, surveys of WP7 Partners, and consultation of key people were used to obtain information on the AEG mechanisms used by twenty-three countries (twenty European countries, United States, Canada [Ontario], and Australia). Results: Interest in the implementation of AEG policies, particularly at the coverage decision stage, is growing. An overview of national experiences was used to draw up a generally applicable five-step policy framework for AEG mechanisms that comprised (i) a first assessment identifying knowledge gaps; (ii) a decision conditional to evidence generation; (iii) generation of the evidence requested; (iv) re-assessment integrating the new evidence; (v) a revised decision. The critical factors for success that were identified were coordination, methodological guidance, funding, and a regulatory framework. Countries were categorized on the basis of current implementation of the proposed policy framework.

Conclusions: International collaboration is necessary to gather a critical mass of high-quality data quickly and to ensure timely access to new promising technologies. The overview produced by WP7A has led to development of tools to facilitate collaboration on evidence generation.

Keywords: Health technology assessment, Evidence-based decision making, Conditional coverage, Conditional marketing approval

Access to new health technologies is subject to many constraints in most developed countries because these technologies are often costly and their impact on health and the healthcare system is uncertain. Obtaining marketing approval for

\footnotetext{
This study was undertaken within the framework of the EUnetHTA Project, which was supported by a grant from the European Commission (Agreement number 2005110 project 790621 ). The sole responsibility for the content of this article (publication, presentation, etc.) lies with the authors, and the European Commission is not responsible for any use that may be made of the information contained therein. All other acknowledgements can be found elsewhere (34)
}

a health product (medicine or medical device) requires, in addition to quality assurance data, evidence on safety and efficacy, mostly collected in controlled settings and according to clearly defined standards (e.g., randomized controlled trials, RCT). However, to obtain coverage or funding for health technologies (medicines, medical devices, and diagnostic, medical, and surgical procedures), evidence on clinical effectiveness is often mandatory and may even need to be substantial. Additional data on quality of life, cost-effectiveness, and impact (e.g., on organization of care) may also be requested. All this further evidence is often collected in real-world, 
pragmatic studies that might not be in the standard form of an RCT and for which no international guidance exists (36).

A major obstacle to ensuring timely access to new health technologies is inadequate evidence on which to base the decision to market or provide coverage, especially if the technology is highly innovative or "promising" (36). Manufacturers, clinicians, and patient groups put pressure on decision makers. They demand early decisions and rapid access, but this increases the risk of inappropriate decisions. The authorities may unduly delay potential benefits to patients by waiting for stronger evidence, or may endorse technologies that later turn out to have a low benefit-risk ratio, to be ineffective, cost-ineffective, or even harmful (36).

Hence, Several countries have therefore developed policy frameworks and mechanisms that allow temporary access to promising technologies while concurrently requesting the generation of additional evidence to reduce uncertainty. We refer to these mechanisms as access with evidence generation (AEG). Their objective is an optimal trade-off between stakeholders' needs, flexibility, responsiveness, and rigor. The decision to provide access is revised when the new evidence becomes available $(33 ; 35)$.

\section{OBJECTIVES}

The aim was to identify the AEG mechanisms implemented in various countries, to use them to draw up a common policy framework applicable at both the marketing approval and coverage decision stages, and to identify the key factors for its successful operation.

\section{METHODS}

\section{Development of the Common Policy Framework}

A draft version of the common policy framework was developed on the basis of a review of the literature on AEG mechanisms and a survey of WP7A Partners (Supplementary Table 1, which is available at www.journals. cambridge.org/thc2009003). A search was made for publications on AEG mechanisms (Databases: MEDLINE, BIOSIS Previews, Current Contents, EMBASE, INAHTA and DARE; period: 1990-2008; languages: English or French) using four systematic search strategies (18). Additional information was obtained from the gray literature (reports on the Web sites of medicines agencies, HTA agencies and national health insurance bodies). The survey attempted to identify the AEG mechanisms in use in Europe (response rate: 93\%). This draft was discussed among WP7A partners, after attending key conferences and interviewing people working in the field. It was amended in the light of the comments. A second survey was performed to identify those countries that implemented the proposed policy framework in part or in full (response rate: $34 \%$ ).

\section{Excluded Topics}

The following were excluded:

(i) Early warning and horizon scanning systems: Their purpose is to identify and inform policy makers on forthcoming new health technologies and to help prioritize HTA. There is no prospective data collection (19).

(ii) Investigational use of nonapproved medical devices or medicines with data collected in clinical trials requested by the regulatory bodies and funded by the applicant: This was considered to be part of the conventional procedure for obtaining marketing approval or licensing, for example, Australia (5) and the United States (22).

(iii) Compassionate use of health technologies: Specific groups of individuals (e.g., with rare diseases) can often obtain rapid access to innovative technologies. This does not, however, usually require data collection $(3 ; 40 ; 49)$.

(iv) Special authorization for use of unapproved medicines: This provides patients with temporary access to medicines that are not yet available in the country, or that are still under development, that is, before marketing authorization. It may be granted in France (Temporary Authorization for use- "Cohort ATU") (1) and Italy (Uso terapeutico di medicinale sottoposoto a sperimentazione clinica) (40) for medicines used to treat serious or rare diseases for which alternative treatments are not available and for which preliminary evidence strongly suggests a positive benefit/risk ratio. This mechanism focuses more on enabling early access than on collecting evidence and cannot replace investigational clinical trials.

(v) "Routine" vigilance systems (15) for medicines and devices: These are based on spontaneous reporting of data. Data collection is neither systematic nor comprehensive (36).

\section{RESULTS}

AEG mechanisms are used when two important decisions are made during a technology's life cycle: (i) marketing approval and (ii) coverage.

\section{AEG Mechanisms Associated With Marketing Approval Decisions}

Medicines. We identified not only the AEG mechanisms recommended by the European Medicines Agency (EMEA), applicable to European Union (EU) countries and implemented by the European Commission (EC), but also country-specific mechanisms (in fourteen of twenty-three countries: Australia, Belgium, Canada, Denmark, Finland, France, Germany, Italy, Latvia, Netherlands, Portugal, Spain, United States, and United Kingdom). Overall, they fell into two categories: (i) conditional marketing authorization and (ii) postmarketing studies (Supplementary Table 2, which is available at www.journals.cambridge.org/thc2009003).

Conditional Marketing Authorization. The EC may grant conditional marketing authorization when the 
new medicines have orphan status, or are intended for seriously debilitating, life-threatening diseases or emergency situations (e.g., pandemics). At least four conditions must be met: (i) preliminary evidence should indicate a positive benefit/risk ratio; (ii) the applicant should be able to provide comprehensive data; (iii) unmet medical needs should be fulfilled; (iv) the public health benefit of immediate access should outweigh the risk due to uncertainty. The decision is made before comprehensive clinical data becomes available. Authorization is granted on a yearly basis and carries the legal obligation to provide further evidence on safety and efficacy (completion or initiation of studies). Conventional marketing authorization may be granted after yearly review of the evidence generated (16).

Some countries also have their own conditional marketing authorization mechanisms, for example, Italy (Autorizzazione subordinata a condizioni) (41), Spain (Autorizacion especial) (3), Denmark (37), Germany (20), Belgium (46), and Canada (Notice of compliance with conditions: NOC/c) (50).

Postmarketing Studies (Including Active Pharmacovigilance). Postmarketing studies are not a prerequisite to marketing approval, but the data collected (e.g., on safety or efficacy in a given population in the usual clinical setting) may impact at any time on the benefit/risk ratio and thus result in changes to marketing authorization (Supplementary Table 2) (17). Most postmarketing studies address safety concerns, as data on safety tends to be limited when approval is granted. Proactive actions to complement routine pharmacovigilance systems (spontaneous reporting of adverse events) are now implemented worldwide.

In the EU, whenever safety concerns arise during clinical trial assessments, the EMEA requests further data collection and appropriate pharmacovigilance, with quantification of adverse events (15). Some Member States implement additional active surveillance to meet their own specific needs: Belgium (46), Finland (44), France (2), Germany (20), Italy (41), Latvia (29), Netherlands (11), Portugal (33), Spain (39), and the United Kingdom (21). This also occurs in Australia (6) and the United States (23).

Medical Devices. Much less information was found on medical devices than on medicines. Two types of AEG mechanisms for marketing approval were identified in seven of twenty-three countries (Australia, Canada, Latvia, Spain, Switzerland, United States, and United Kingdom): (i) conditional licensing and (ii) postmarketing clinical follow-up.

Conditional Licensing. Conditional licensing may be granted to new moderate- or high-risk medical devices in Canada "when there is reasonable assurance that the device is safe and effective but supplemental information is required to support this conclusion" (49). The applicant has to fund and set up studies to collect additional clinical data to confirm the benefit/risk ratio within a set deadline (49).
Postmarketing Clinical Follow-up (or Postapproval Surveillance). Several follow-up methods are available $(24 ; 28 ; 56)$ : long-term surveillance of the patients who were included in preapproval clinical trials, prospective observational studies, registries, or new clinical trials. The Global Harmonization Task Force (GHTF) has performed extensive work on regulatory approaches about postmarketing surveillance and clinical assessment of medical devices and has proposed guidance $(25 ; 26)$. Funding comes from either the holder of the marketing approval or public institutions.

A new EU directive applicable from March 2010 (13) will request systematic data collection during postmarketing surveillance (unless nonapplicability can be justified). This follows the guidance issued by the Medical Devices Evaluation Committee (MEDDEV) (14) - already implemented by Latvia (29) and Switzerland (52).

\section{AEG Mechanisms Associated With Coverage Decisions}

AEG mechanisms associated with coverage decisions, unlike those associated with marketing approval are recent. To date, few countries have implemented such mechanisms $(10 ; 32 ; 45 ; 55)$, but an increasing number are showing interest and attempting to identify mechanisms that will meet their local needs and constraints. These mechanisms are applied to medicines, medical devices, and/or procedures (Table 1). We identified only twelve of twenty-three countries implementing AEG mechanisms before coverage decisions: Canada (Ontario) $(42 ; 43 ; 47)$, Spain (38), Australia (43), United States (55), Switzerland (12), Sweden (53), Belgium (9), Netherlands (51), France (27), England/Wales (10;45), Germany (10), and Italy (4).

The mechanisms fell into three main categories of uncertainty: (i) the "No, unless ..." category which considers that evidence is inadequate to grant coverage unless additional requirements are met; (ii) the "Yes, but..." category which considers that the evidence is reasonably adequate to grant coverage provided that additional evidence is generated, and (iii) the "Yes for now" category which considers that the evidence is adequate to grant standard coverage but requests data on specific points (e.g., on conditions of use). Access may be limited to patients included in a clinical trial or treated in data collection centers, or may be unrestricted. Tables 1 and 2 show the AEG mechanisms implemented by each category and the bodies involved.

Some countries (Canada [Ontario], Spain, Australia, United States, Switzerland, Sweden, Belgium, Netherlands, France, and England/Wales) implement a structured form of AEG (conditional coverage) which is usually part of an established policy framework, in which the initial decision on coverage is conditional to the generation of evidence in response to the decision-makers' requests $(10 ; 32 ; 55)$. Data are collected prospectively under experimental conditions 
Table 1. Main Characteristics of AEG Mechanisms Associated with Coverage Decisions

\begin{tabular}{|c|c|c|c|c|}
\hline $\begin{array}{l}\text { AEG system (Name, } \\
\text { country) }\end{array}$ & $\begin{array}{l}\text { Health technologies } \\
\text { concerned }\end{array}$ & $\begin{array}{l}\text { Type of initial } \\
\text { decision }\end{array}$ & $\begin{array}{l}\text { Requirement for AEG } \\
\text { decision }\end{array}$ & Examples \\
\hline $\begin{array}{l}\text { Only in research } \\
\text { (England/Wales) }\end{array}$ & $\begin{array}{l}\text { Medicines, medical } \\
\text { devices, interventional } \\
\text { procedures, and public } \\
\text { health interventions }\end{array}$ & $\begin{array}{l}\text { "No, } \\
\text { unless. .." }\end{array}$ & $\begin{array}{l}\text { The use of a promising } \\
\text { technology or a public } \\
\text { health intervention is not } \\
\text { supported by enough } \\
\text { robust evidence }\end{array}$ & $\begin{array}{l}\text { - Laparoscopic surgery for } \\
\text { colorectal cancer } \\
\text { - Taxanes as adjuvant in } \\
\text { early node positive breast } \\
\text { cancer } \\
\text { - Verteporfin in ARMD... }\end{array}$ \\
\hline $\begin{array}{l}\text { Suspended coverage } \\
\text { decision with pilot } \\
\text { project (Germany) }\end{array}$ & $\begin{array}{l}\text { Medical devices within a } \\
\text { procedure }\end{array}$ & $\begin{array}{l}\text { "No, } \\
\text { unless..." }\end{array}$ & $\begin{array}{l}\text { Not enough evidence to } \\
\text { conclude on the benefit, } \\
\text { necessity, or efficiency }\end{array}$ & $\begin{array}{l}\text { - Acupuncture for chronic } \\
\text { pain } \\
\text { - Screening for skin cancer } \\
\text { - Balneo-phototherapy... }\end{array}$ \\
\hline $\begin{array}{l}\text { Conditionally funded field } \\
\text { evaluation (Canada, } \\
\text { Ontario) }\end{array}$ & $\begin{array}{l}\text { Medicines, medical } \\
\text { devices, procedures, and } \\
\text { public health } \\
\text { interventions }\end{array}$ & "Yes, but. .." & $\begin{array}{l}\text { - Uncertainty (low quality } \\
\text { of evidence) about } \\
\text { effectiveness, } \\
\text { cost-effectiveness, } \\
\text { or safety } \\
\text { - Need for quality controls } \\
\text { prior to unrestricted } \\
\text { diffusion } \\
\text { - Potential disruptive } \\
\text { effects } \\
\text { - Large potential } \\
\text { investment }\end{array}$ & $\begin{array}{l}\text { - PET scanners } \\
\text { - Endovascular treatment of } \\
\text { abdominal aneurysms } \\
\text { - Drug eluting stents } \\
\text { - Surgical treatment of } \\
\text { epilepsy } \\
\text { - Cardiac CT } \\
\text { angiography... }\end{array}$ \\
\hline Monitored use (Spain) & $\begin{array}{l}\text { Medical devices, medical } \\
\text { and surgical procedures }\end{array}$ & "Yes, but. .." & $\begin{array}{l}\text { Uncertainty about } \\
\text { effectiveness and safety } \\
\text { at the initial coverage } \\
\text { decision stage }\end{array}$ & $\begin{array}{l}\text { - Surgical treatment of } \\
\text { epilepsy } \\
\text { - PET scanners } \\
\text { - Endovascular treatment of } \\
\text { abdominal aneurysms... }\end{array}$ \\
\hline Interim funding (Australia) & $\begin{array}{l}\text { Medical devices, medical } \\
\text { and surgical procedures }\end{array}$ & "Yes, but. .." & $\begin{array}{l}\text { For promising technologies } \\
\text { which are (i) safe, } \\
\text { effective but with } \\
\text { uncertain } \\
\text { cost-effectiveness or (ii) } \\
\text { with uncertain } \\
\text { effectiveness and safety } \\
\text { but potent } \\
\text { cost-effectiveness }\end{array}$ & $\begin{array}{l}\text { - PET scanners } \\
\text { - Deep-brain stimulators } \\
\text { - Endovascular treatment of } \\
\text { abdominal aneurysms. . . }\end{array}$ \\
\hline $\begin{array}{l}\text { Coverage with evidence } \\
\text { development (CMS) } \\
\text { (US) }\end{array}$ & $\begin{array}{l}\text { Medicines, medical } \\
\text { devices, medical and } \\
\text { surgical procedures }\end{array}$ & "Yes, but. .." & $\begin{array}{l}\text { Evidence complementary } \\
\text { to existing medical } \\
\text { evidence is required on } \\
\text { effectiveness, safety or } \\
\text { cost-effectiveness }\end{array}$ & $\begin{array}{l}\text { - Lung volume reduction } \\
\text { surgery } \\
\text { - Cochlear implants } \\
\text { - Implantable cardioverter } \\
\text { defibrillators } \\
\text { - PET scanners. . . }\end{array}$ \\
\hline $\begin{array}{l}\text { Medical service under } \\
\text { evaluation (Switzerland) }\end{array}$ & Controversial procedures & "Yes, but. .." & $\begin{array}{l}\text { Uncertainty about } \\
\text { effectiveness and safety } \\
\text { at the initial coverage } \\
\text { decision stage }\end{array}$ & $\begin{array}{l}\text { - Bariatric surgery } \\
\text { - Surgical treatment of } \\
\text { epilepsy } \\
\text { - Curietherapy in prostate } \\
\text { cancer } \\
\text { - Intervertebral disc } \\
\text { replacement } \\
\text { - Verteporfin in ARMD... }\end{array}$ \\
\hline $\begin{array}{l}\text { Reimbursement with } \\
\text { conditions (Sweden) }\end{array}$ & Innovative medicines & "Yes, but. .." & $\begin{array}{l}\text { Uncertainty about } \\
\text { effectiveness, safety, } \\
\text { cost-effectiveness, or } \\
\text { conditions of use }\end{array}$ & $\begin{array}{l}\text { - Diabetes and weight loss } \\
\text { treatment } \\
\text { - Cancer drugs and } \\
\text { biologicals... }\end{array}$ \\
\hline $\begin{array}{l}\text { Conditional reimbursement } \\
\text { (Belgium) }\end{array}$ & Innovative implants & "Yes, but. .." & $\begin{array}{l}\text { Uncertainty about } \\
\text { effectiveness, safety, or } \\
\text { condition of use }\end{array}$ & $\begin{array}{l}\text { - Deep brain stimulation } \\
\text { - Endovascular treatment of } \\
\text { abdominal aneurysms } \\
\text { - Drug eluting stents for } \\
\text { diabetic patients } \\
\text { - Contralateral cochlear } \\
\text { implant. . }\end{array}$ \\
\hline
\end{tabular}


Carbonneil et al.

Table 1. Continued

\begin{tabular}{|c|c|c|c|c|}
\hline $\begin{array}{l}\text { AEG system (Name, } \\
\text { country) }\end{array}$ & $\begin{array}{l}\text { Health technologies } \\
\text { concerned }\end{array}$ & $\begin{array}{l}\text { Type of initial } \\
\text { decision }\end{array}$ & $\begin{array}{l}\text { Requirement for AEG } \\
\text { decision }\end{array}$ & Examples \\
\hline $\begin{array}{l}\text { Conditional reimbursement } \\
\text { (Netherlands) }\end{array}$ & $\begin{array}{l}\text { Hospital prescribed } \\
\text { medicines (e.g., costly or } \\
\text { orphan medicines) }\end{array}$ & "Yes, but..." & $\begin{array}{l}\text { Uncertainty regarding } \\
\text { relative therapeutic } \\
\text { value, relative } \\
\text { cost-effectiveness, and } \\
\text { importance to public } \\
\text { health }\end{array}$ & $\begin{array}{l}\text {-Bevacizumab, } \\
\text {-Trastuzumab, } \\
\text {-Anidulafungin. ... }\end{array}$ \\
\hline $\begin{array}{l}\text { Still in clinical research } \\
\text { (France) }\end{array}$ & $\begin{array}{l}\text { Medical and surgical } \\
\text { procedures }\end{array}$ & "Yes, but. .." & $\begin{array}{l}\text { Clinical benefit cannot be } \\
\text { fully established }\end{array}$ & $\begin{array}{l}\text { - Intensity-modulated } \\
\text { radiation therapy } \\
\text { - Extracranial stereotactic } \\
\text { radiotherapy } \\
\text { - Biochemical markers of } \\
\text { liver fibrosis }{ }^{\mathrm{a}} . .\end{array}$ \\
\hline $\begin{array}{l}\text { Post-listing studies } \\
\text { (France) }\end{array}$ & $\begin{array}{l}\text { Medicines and medical } \\
\text { devices }\end{array}$ & $\begin{array}{l}\text { "Yes for } \\
\text { now..." }\end{array}$ & $\begin{array}{l}\text { Uncertainty on conditions } \\
\text { of use, safety, impact on } \\
\text { organization of } \\
\text { healthcare... }\end{array}$ & $\begin{array}{l}\text { - IFN- } \beta \\
\text { - Anti TNF- } \alpha \text { medicines } \\
\text { - Transcutaneous implanted } \\
\text { pulmonary valve. . }\end{array}$ \\
\hline $\begin{array}{l}\text { Independent research on } \\
\text { medicines (Italy) }\end{array}$ & $\begin{array}{l}\text { Medicines, public health } \\
\text { interventions }\end{array}$ & $\begin{array}{l}\text { "Yes for } \\
\text { now..." }\end{array}$ & $\begin{array}{l}\text { - Rare diseases; } \\
\text { - High impact in terms of } \\
\text { public health or economy; } \\
\text { - Long-term safety during } \\
\text { treatment of chronic } \\
\text { diseases... }\end{array}$ & $\begin{array}{l}\text { - IFN- } \beta \text { vs azathioprine } \\
\text { - Bevacizumab } \\
\text { - Evaluation of impact of } \\
\text { educational interventions } \\
\text { of patient with } \\
\text { psoriasis... }\end{array}$ \\
\hline
\end{tabular}

${ }^{a}$ Recommended by national HTA agency, but no data generated.

AEG, Access with Evidence Generation; ARMD, age-related macular degeneration; PET, positron emission tomography; CT, computed tomography; IFN, interferon; TNF, tumor necrosis factor.

(clinical trials) or under real-life conditions (registries, observational, pragmatic, or health economics studies). Their results are taken into account in the reassessment and in the subsequent revised coverage decision $(10 ; 32 ; 55)$ that may lead to standard coverage, modification of coverage conditions, or even to delisting.

Table 3 compares the strengths and weaknesses of all these AEG mechanisms.

\section{Common Policy Framework for AEG Mechanisms}

The above descriptions of the AEG mechanisms currently implemented, whether for marketing approval or coverage/reimbursement decisions, were used to construct a common-denominator model underlying a five-step policy framework (Supplementary Figure 1, which is available at www.journals.cambridge.org/thc2009003):

Step 1. A first assessment pinpoints evidence gaps and data needs and proposes a plan for data collection (type of data and study, time period, etc.).

Step 2. A decision is made on conditional and temporary access to the technology. This decision is based on the first assessment and is accompanied by a request for evidence generation (which type of data needs to be collected and analyzed to fill which evidence gaps, in reply to which uncertainties voiced by the decision makers).
Step 3. During an interim period of conditional access to the technology, the data that have been requested are collected and the use of the technology is monitored. Conditions of use are usually restricted and well-defined (e.g., in a limited number of centers, performed by highly skilled professionals, etc.).

Step 4. A second assessment that includes the new evidence is performed.

Step 5. A revised decision based on this second assessment is made with regard to access to the technology.

Outcomes of this process may be widespread and appropriate availability of the technology, restricted diffusion, or discontinuation of use.

During construction of the model, WP7A Partners drew attention to the reported barriers against establishing and operating a completely operational system at the coverage stage $(10 ; 32 ; 55)$ and stressed the need to establish critical success factors (Box 1).

This common policy framework and associated critical success factors apply to the implementation of AEG both at the marketing approval and coverage decision stages. It is derived from the current regulatory framework for medicines. Although all five steps and all factors are usually applied in the case of medicines (EMEA has defined study designs, quality requirements, organized the coordination of bodies, etc.), many steps and factors are often omitted in the case of coverage decisions (Table 3 ). 
Table 2. Role of Identified Collaborators in AEG Mechanisms Associated with Coverage Decisions

\begin{tabular}{|c|c|c|c|c|c|}
\hline $\begin{array}{l}\text { AEG system (Name, } \\
\text { country) }\end{array}$ & $\begin{array}{l}\text { Coordinating } \\
\text { structure }\end{array}$ & $\begin{array}{l}\text { Source of funding for } \\
\text { data collection }\end{array}$ & $\begin{array}{l}\text { Structure performing } \\
\text { data collection }\end{array}$ & $\begin{array}{c}\text { Structure performing } \\
\text { data analysis }\end{array}$ & $\begin{array}{l}\text { Decision-making } \\
\text { authority }\end{array}$ \\
\hline $\begin{array}{l}\text { Only in research } \\
\text { (England/Wales) }\end{array}$ & NICE & $\begin{array}{l}\text {-Public (NHS R\&D, } \\
\text { MRC...) } \\
\text {-Private } \\
\quad \text { (manufacturers...) }\end{array}$ & $\begin{array}{l}\text { Various partners } \\
\text { (health } \\
\text { professionals, } \\
\text { manufacturers...) }\end{array}$ & $\begin{array}{l}\text { Various partners } \\
\text { (health } \\
\text { professionals, } \\
\text { manufacturers...) }\end{array}$ & NICE \\
\hline $\begin{array}{l}\text { Suspended coverage } \\
\text { decision with pilot } \\
\text { project (Germany) }\end{array}$ & $\begin{array}{l}\text { Statutory Health } \\
\text { Insurance/ } \\
\text { Association of } \\
\text { statutory health } \\
\text { physicians }\end{array}$ & $\begin{array}{l}\text { - Public (Statutory } \\
\text { Health Insurance) }\end{array}$ & $\begin{array}{l}\text { Health professionals } \\
\text { (mostly the } \\
\text { Association of } \\
\text { statutory health } \\
\text { physicians) }\end{array}$ & Not determined & GB-A \\
\hline $\begin{array}{l}\text { Conditionally funded } \\
\text { field evaluation } \\
\text { (Canada, Ontario) }\end{array}$ & OHTAC/MAS & $\begin{array}{l}\text { Public (Ministry of } \\
\text { Health and } \\
\text { long-term care) }\end{array}$ & $\begin{array}{l}\text { PATH, THETA, other } \\
\text { academic partners, } \\
\text { research } \\
\text { organizations }\end{array}$ & $\begin{array}{l}\text { PATH, THETA, other } \\
\text { academic partners }\end{array}$ & $\begin{array}{l}\text { Ministry of Health } \\
\text { and long-term care }\end{array}$ \\
\hline $\begin{array}{l}\text { Monitored use } \\
\text { (Spain) }\end{array}$ & $\begin{array}{l}\text { National Health } \\
\text { Service's } \\
\text { Interterritorial } \\
\text { Council }\end{array}$ & $\begin{array}{l}\text { Public (Ministry of } \\
\text { Health) }\end{array}$ & Public hospitals & $\begin{array}{l}\text { HTA agencies } \\
\text { (AETS, AETSA, } \\
\text { Osteba, } \\
\text { Avalia-t. ..) }\end{array}$ & Ministry of Health \\
\hline $\begin{array}{l}\text { Interim funding } \\
\text { (Australia) }\end{array}$ & MSAC & $\begin{array}{l}\text { Public (Ministry of } \\
\text { Health and aging) }\end{array}$ & $\begin{array}{l}\text { Associations } \\
\text { of health } \\
\text { professionals }\end{array}$ & $\begin{array}{l}\text { Associations of } \\
\text { health } \\
\text { professionals }\end{array}$ & $\begin{array}{l}\text { Ministry of Health } \\
\text { and aging }\end{array}$ \\
\hline $\begin{array}{l}\text { Coverage with } \\
\text { evidence } \\
\text { development } \\
\text { (CMS) (US) }\end{array}$ & CMS & $\begin{array}{l}\text { Mostly public (CMS } \\
\text { for clinical costs } \\
\text { only, stakeholders } \\
\text { for other costs. . ) }\end{array}$ & $\begin{array}{l}\text { Various partners } \\
\text { (institutions, health } \\
\text { professionals...) }\end{array}$ & $\begin{array}{l}\text { Various partners } \\
\text { (institutions, health } \\
\text { professionals...) }\end{array}$ & CMS \\
\hline $\begin{array}{l}\text { Medical service } \\
\text { under evaluation } \\
\text { (Switzerland) }\end{array}$ & $\begin{array}{l}\text { Federal Office of } \\
\text { Public Health }\end{array}$ & $\begin{array}{l}\text { Mostly public } \\
\text { (Sickness Funds or } \\
\text { applicants) }\end{array}$ & $\begin{array}{l}\text { Applicant (health } \\
\text { professionals, } \\
\text { manufacturers) }\end{array}$ & Not determined & $\begin{array}{l}\text { Federal Department } \\
\text { of Home Affairs } \\
\text { (advised by ELK) }\end{array}$ \\
\hline $\begin{array}{l}\text { Reimbursement with } \\
\text { conditions } \\
\text { (Sweden) }\end{array}$ & TLV & Manufacturers & Manufacturers & TLV & TLV \\
\hline $\begin{array}{l}\text { Conditional } \\
\text { reimbursement } \\
\text { (Belgium) }\end{array}$ & KCE/INAMI & Public (INAMI) & Health professionals & KCE/INAMI & INAMI \\
\hline $\begin{array}{l}\text { Conditional } \\
\text { reimbursement } \\
\text { (Netherlands) }\end{array}$ & CVZ & $\begin{array}{l}\text { Manufacturers/ } \\
\text { ZonMW }\end{array}$ & $\begin{array}{l}\text { Various partners } \\
\text { (Manufacturers, } \\
\text { health } \\
\text { professionals, } \\
\text { academic partners, } \\
\text { institutions...) }\end{array}$ & CVZ & $\begin{array}{l}\text { Ministry of Health, } \\
\text { Welfare and Sport }\end{array}$ \\
\hline $\begin{array}{l}\text { Still in clinical } \\
\text { research (France) }\end{array}$ & HAS & $\begin{array}{l}\text { UNCAM for clinical } \\
\text { costs only; } \\
\text { currently no } \\
\text { funding for other } \\
\text { costs }\end{array}$ & Health professionals & HAS & HAS/UNCAM \\
\hline $\begin{array}{l}\text { Post-listing studies } \\
\text { (France) }\end{array}$ & HAS & Manufacturers & Manufacturers & Manufacturers & Ministry of Health \\
\hline $\begin{array}{l}\text { Independent research } \\
\text { on medicines } \\
\text { (Italy) }\end{array}$ & AIFA & Public (AIFA) & Health professionals & AIFA & Regional institutions \\
\hline \multicolumn{6}{|c|}{ 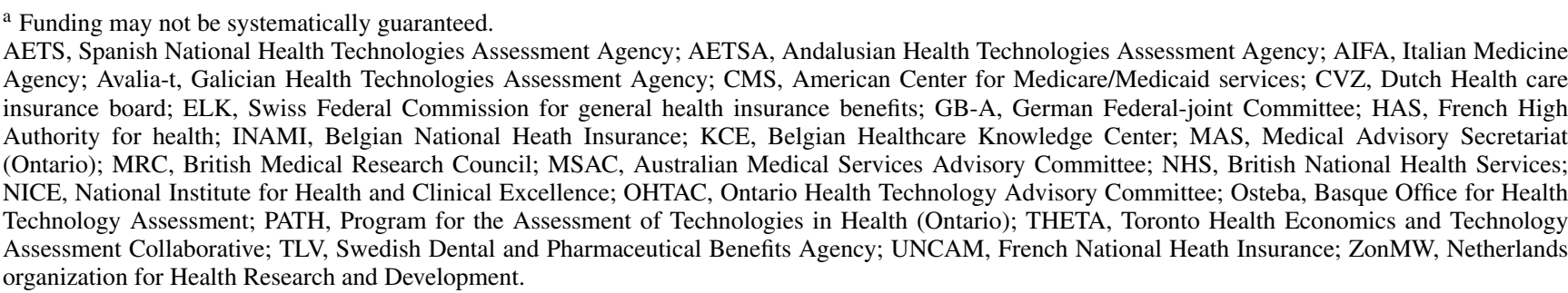 } \\
\hline
\end{tabular}


Carbonneil et al.

Table 3. Reported Strengths and Weaknesses of AEG Systems at the Coverage Stage

\begin{tabular}{|c|c|c|}
\hline AEG system (Name, country) & Reported strengths & Reported weaknesses \\
\hline $\begin{array}{l}\text { Only in research } \\
\text { (England/Wales) }\end{array}$ & $\begin{array}{l}\text { - Regulatory framework } \\
\text { - Methodological guidance }\end{array}$ & $\begin{array}{l}\text { - No dedicated funding } \\
\text { - No systematic collaboration between partners } \\
\text { - Not a systematic process (opportunistic) }\end{array}$ \\
\hline $\begin{array}{l}\text { Suspended coverage decision } \\
\text { with pilot project (Germany) }\end{array}$ & - Dedicated funding ${ }^{\mathrm{a}}$ & $\begin{array}{l}\text { - Incomplete regulatory framework as regards project } \\
\text { implementation and use of results in decision making } \\
\text { - For medical devices used as part of a procedure only } \\
\text { - No methodological guidance } \\
\text { - No systematic collaboration }\end{array}$ \\
\hline $\begin{array}{l}\text { Conditionally funded field } \\
\text { evaluation (Canada, Ontario) }\end{array}$ & $\begin{array}{l}\text { - Dedicated funding } \\
\text { - Systematic collaboration between partners } \\
\text { - Regulatory framework } \\
\text { - Methodological guidance } \\
\text { - Operational system } \\
\text { - Great way to engage end users. }\end{array}$ & $\begin{array}{l}\text { - High pressure } \\
\text { - Regional system (limited to Ontario) }\end{array}$ \\
\hline Monitored use (Spain) & $\begin{array}{l}\text { - Dedicated funding } \\
\text { - Systematic collaboration between partners } \\
\text { - Regulatory framework } \\
\text { - Methodological guidance } \\
\text { - Operational system }\end{array}$ & - No selection criteria for technologies to be monitored \\
\hline Interim funding (Australia) & $\begin{array}{l}\text { - Dedicated funding } \\
\text { - Regulatory framework } \\
\text { - Methodological guidance } \\
\text { - Operational system. }\end{array}$ & $\begin{array}{l}\text { - For medical devices and procedures only } \\
\text { - No systematic collaboration between partners } \\
\text { - Funding (MBS) not fully adapted to "interim funding" } \\
\text { - Trial duration too long ( }>3 \text { yr) for conditional } \\
\text { coverage } \\
\text { - National target populations too small; interim funding } \\
\text { while awaiting results of international studies }\end{array}$ \\
\hline $\begin{array}{l}\text { Coverage with evidence } \\
\text { development (CMS) (US) }\end{array}$ & $\begin{array}{l}\text { - Regulatory framework } \\
\text { - Methodological guidance (partial) }\end{array}$ & $\begin{array}{l}\text { - No dedicated global funding } \\
\text { - No systematic collaboration between partners } \\
\text { - Not a systematic process (opportunistic) } \\
\text { - Difficulties in designing CED studies }\end{array}$ \\
\hline $\begin{array}{l}\text { Medical service under evaluation } \\
\text { (Switzerland) }\end{array}$ & $\begin{array}{l}\text { - Regulatory framework } \\
\text { - Dedicated funding } \\
\text { - Operational system }\end{array}$ & $\begin{array}{l}\text { - For controversial medical procedures only } \\
\text { - No systematic collaboration (depends on applicant) } \\
\text { - Constraints of public administration human and } \\
\text { financial resources }\end{array}$ \\
\hline $\begin{array}{l}\text { Reimbursement with conditions } \\
\text { (Sweden) }\end{array}$ & $\begin{array}{l}\text { - Regulatory framework } \\
\text { - Dedicated funding }{ }^{\mathrm{a}} \\
\text { - Mandatory engagement of manufacturer } \\
\text { - Operational system }\end{array}$ & $\begin{array}{l}\text { - For innovative drugs only } \\
\text { - Difficulties in interpreting observational studies } \\
\text { - Difficulties with effectiveness studies }\end{array}$ \\
\hline $\begin{array}{l}\text { Conditional reimbursement } \\
\text { (Belgium) }\end{array}$ & $\begin{array}{l}\text { - Regulatory framework }{ }^{\mathrm{b}} \\
\text { - Dedicated funding } \\
\text { - Collaboration between partners } \\
\text { - Operational system }\end{array}$ & - For innovative new implants only \\
\hline $\begin{array}{l}\text { Conditional reimbursement } \\
\text { (Netherlands) }\end{array}$ & $\begin{array}{l}\text { - Regulatory framework } \\
\text { - Dedicated funding } \\
\text { - Mandatory engagement of manufacturer }\end{array}$ & $\begin{array}{l}\text { - For hospital-prescribed medicines (costly or orphan } \\
\text { medicines) only }\end{array}$ \\
\hline Still in clinical research (France) & - Regulatory framework ${ }^{\mathrm{b}}$ & $\begin{array}{l}\text { - For medical and surgical procedures only } \\
\text { - No dedicated global funding } \\
\text { - No collaboration between partners } \\
\text { - No operational system. }\end{array}$ \\
\hline Post-listing studies (France) & $\begin{array}{l}\text { - Dedicated funding }{ }^{\mathrm{a}} \text { (by the manufacturers) } \\
\text { - Regulatory framework } \\
\text { - Methodological guidance } \\
\text { - Mandatory engagement of manufacturer }\end{array}$ & $\begin{array}{l}\text { - For medicines and medical devices only } \\
\text { - Difficulties to find agreement on study design } \\
\text { - No conditional or temporary coverage (but linked } \\
\text { with strict conditions). }\end{array}$ \\
\hline $\begin{array}{l}\text { Independent research on } \\
\text { medicines (Italy) }\end{array}$ & $\begin{array}{l}\text { - Implemented regulatory framework } \\
\text { - Dedicated funding }{ }^{\mathrm{a}} \\
\text { - Methodological guidance }\end{array}$ & $\begin{array}{l}\text { - For medicines only } \\
\text { - No systematic collaboration (research projects only) } \\
\text { - No conditional or temporary coverage }\end{array}$ \\
\hline
\end{tabular}

${ }^{a}$ Dedicated funding may not be systematically guaranteed.

${ }^{\mathrm{b}} \mathrm{A}$ change in the law on innovative technologies has been proposed to achieve a more operational mechanism. 
Table 4. Degree of Implementation of AEG Mechanisms by Various Countries

\begin{tabular}{|c|c|c|c|c|c|}
\hline \multirow[b]{2}{*}{ Country } & \multicolumn{2}{|c|}{ Marketing approval } & \multicolumn{3}{|c|}{ Coverage decision } \\
\hline & Medicine & Medical device & Medicine & Medical device & Procedure \\
\hline Canada (Ontario) & $+++^{\mathrm{N}}$ & +++ & +++ & +++ & +++ \\
\hline Spain & $+++{ }^{\mathrm{E}, \mathrm{N}}$ & +++ & +++ & +++ & +++ \\
\hline Australia & $+++{ }^{N}$ & +++ & $+++/++$ & +++ & +++ \\
\hline US & $+++{ }^{N}$ & +++ & +++ & +++ & +++ \\
\hline England/Wales & $+++{ }^{\mathrm{E}, \mathrm{N}}$ & ++ & +++ & +++ & +++ \\
\hline France & $+++{ }^{\mathrm{E}, \mathrm{N}}$ & ++ & +++ & +++ & $++/+$ \\
\hline Germany & $+++{ }^{\mathrm{E}, \mathrm{N}}$ & ++ & ++ & $+++/++$ & ++ \\
\hline Sweden & $+++{ }^{E}$ & - & +++ & ++ & ++ \\
\hline Belgium & $+++{ }^{\mathrm{E}, \mathrm{N}}$ & - & - & +++ & - \\
\hline Italy & $+++{ }^{\mathrm{E}, \mathrm{N}}$ & - & $+++/++$ & $+1-$ & $+/-$ \\
\hline Netherlands & $+++{ }^{\mathrm{E}, \mathrm{N}}$ & - & $+++1-$ & - & - \\
\hline Switzerland & - & +++ & - & - & $+++1-$ \\
\hline Austria & $+++{ }^{\mathrm{E}}$ & - & ++ & ++ & ++ \\
\hline Denmark & $+++{ }^{\mathrm{E}, \mathrm{N}}$ & - & ++ & ++ & ++ \\
\hline Latvia & $+++{ }^{\mathrm{E}, \mathrm{N}}$ & +++ & - & - & - \\
\hline Portugal & $+++{ }^{\mathrm{E}, \mathrm{N}}$ & - & - & - & - \\
\hline Finland & $+++{ }^{\mathrm{E}, \mathrm{N}}$ & - & - & - & - \\
\hline Poland & $+++{ }^{\mathrm{E}}$ & - & - & - & - \\
\hline Ireland & $+++{ }^{\mathrm{E}}$ & - & - & - & - \\
\hline Estonia & $+++{ }^{\mathrm{E}}$ & - & - & - & - \\
\hline Slovenia & $+++{ }^{\mathrm{E}}$ & - & - & - & - \\
\hline Cyprus & $+++{ }^{\mathrm{E}}$ & - & - & - & - \\
\hline Norway & - & - & - & - & - \\
\hline
\end{tabular}

Note. +++ , full AEG; ++ , partial AEG; + , passive AEG; - , No AEG. ${ }^{\mathrm{E}}$, AEG implemented by EMEA and applicable in European Countries; ${ }^{\mathrm{N}}$, country-specific AEG implemented at national level.

Box 1. Barriers to and Critical Success Factors for Evidence Generation

Barriers
Critical success factors

- Coordinating body overseeing the contributions and collaboration of all participants

- Scientific leadership and clear guidance on key methodological issues (e.g., study design) for relevant and high-quality evidence

- Dedicated funding for data collection and analysis (e.g., studies and registries), regardless of source

- Regulatory framework

\section{Ranking Implementation of AEG Mechanisms}

We compared our observations on the implementation of AEG mechanisms with the model policy framework. To do this, we arbitrarily defined four levels of execution:

(i) Full implementation: All five steps and all four critical success factors are implemented. The first assessment identifies evidence gaps. Data collection meets quality standards. The revised decision is based on an updated literature review and on the additional data generated.

(ii) Partial implementation: The five steps are fully operational. The first assessment identifies evidence gaps. However, data collection is hampered by national constraints on implementation of the success factors. The revised decision is based on an updated literature review, but on partial data collection only (mostly registry data).

(iii) Passive implementation: The first assessment identifies evidence gaps, but data are not collected usually for financial or regulatory reasons. The revised decision is based on an updated literature review only.

(iv) No implementation: There is no systematic identification of new technologies nor any follow-up of their diffusion. No second assessment is performed.

As illustrated in Table 4, the degree of implementation (full, partial, passive, or absent) varied widely among the twentythree countries. 


\section{DISCUSSION}

Timely access to new promising technologies (marketing approval or coverage) often depends on the generation of additional evidence (36). Access with evidence generation (AEG) is well known in the context of marketing approval, but is a more recent concept in relation to coverage $(10 ; 32 ; 55)$. Few countries have experience of AEG as applied to coverage. The issue has been hotly debated by WP7A and also by the Health Technology Assessment international (HTAi) special interest group on "conditional coverage and evidence development for promising technologies" (30).

WP7A reviewed the national AEG mechanisms associated with coverage decisions using the gray literature (Web sites informing on local laws, regulatory frameworks, and procedures), interviews, and surveys of WP7A Partners, as published data was scarce especially for Europe. The review may have shortcomings, but it has served its purpose of informing a debate among WP7A Partners to move toward international collaboration.

Coverage decisions were linked to AEG mechanisms in ten of the twenty-three countries studied (Canada [Ontario], Spain, Australia, United States, Netherlands, Sweden, Switzerland, England/Wales, France, and Germany) $(10 ; 32 ; 55)$. In most of these countries, requests for AEG filled knowledge gaps and enabled decisions on the appropriate diffusion of several promising technologies after conditional coverage, for example, lung volume reduction surgery (United States), positron emission tomography scanning (Canada, Australia, Spain, United States), and endovascular repair of abdominal aneurysms (Canada, Australia, Spain). However, the system does not always work. For instance, no funding could be found for evidence generation on cochlear implants in the United States, and the lack of meaningful endpoints for implantable cardioverter defibrillators (ICD) meant that American ICD register results were disappointing and of no use (54).

We used the review to construct a five-step model policy framework for AEG mechanisms for implementation and/or adaptation by interested countries. This framework, together with its critical success factors, covers new and existing conditional coverage frameworks and includes relevant adaptations from the long-standing regulatory frameworks for medicines. It is thus applicable to AEG at the marketing approval stage (where all 5 steps are implemented) and at the coverage decision stage (where steps are often omitted). The framework revolved around the collection of relevant data on promising technologies that could effectively support decisions on appropriate diffusion or discontinuation of use. However, the actions needed to generate these data may require changes to currently applicable policy frameworks.

The critical success factors that were identified were (i) coordination, (ii) methodological guidance, (iii) funding, and (iv) an implemented regulatory framework. Their absence can hamper data collection. (i) A named body should coordinate all actions. For instance, in Spain and Canada, decision makers, HTA organizations, healthcare professionals, and researchers cooperate to garner data and implement policy recommendations under the supervision of a coordinating body. In contrast, in the French system of conditional coverage for medical and surgical procedures, coordination between the Ministry of Health, national health insurance, the HTA agency, health professionals, and industry has been poor despite each stakeholder's interest, partly because of the lack of a suitable funding mechanism. Hopefully, a scheduled change in the law on innovative technologies will lead to improvements. In the case of medicines, collaboration is needed between the regulatory setting (assessment and marketing decision) and the HTA setting (assessment and coverage decision) to avoid duplication of work (e.g., between national medicine agencies and HTA agencies). Assessment reports from the marketing authorization process or postmarketing data may also be useful in an initial HTA.

(ii) There must be clear scientific guidance to define precisely the most appropriate type of data and study design to ensure that evidence will ultimately improve. The timeliness and duration of data collection are also important, although there is no consensus on criteria to determine duration $(32 ; 54)$.

(iii) Dedicated financing mechanisms for data collection and analysis are essential, especially before deciding on coverage. Funding should be adequate so that data collection does not end prematurely, or result in generating low-quality data. It could be restricted to data collection in certain centers only. There might be just a single source of funding (generally public) or multiple sources (public, private, or mixed), often each covering a specific cost. For example, the National Heart Lung and Blood Institute (NHLBI) supported the funding and administration of the National Emphysema Treatment Trial (NETT), whereas Centers for Medicare/Medicaid Services (CMS) paid for patient care (8). Opportunity for public-private partnership should also be considered (7).

(iv) A regulatory framework should clearly state the role and responsibility of each Partner and ensure that the AEG results are used during the revised decision process. For instance, in Germany, the results from pilot projects are not systematically considered during reassessment because of lack of a regulatory framework.

Other challenging issues concerning promising health technologies, and not listed among the above success factors are: (i) collaboration with academic research, (ii) uncertainty thresholds, selection and prioritization, (iii) timing of the request for evidence generation, and (iv) the relationship between HTA and AEG.

(i) Decisions on conditional access are usually made independently of decisions on clinical research. We noted that AEG mechanisms were strengthened when HTA agencies, decision makers, and research institutions collaborate, as in Ontario.

(ii) The criteria for estimating uncertainty and prioritizing technologies that might benefit from an AEG mechanism should be explicit, especially as resources are limited $(54 ; 55)$. 
(iii) When an authority should request evidence generation is a moot point. The trend is toward providing scientific advice in the early stages of the technology's development, as some medicines agencies already do.

(iv) The initial HTA should clearly quantify uncertainty, identify knowledge gaps and data needs, and indicate avenues for further research with possibly clear guidance on which data should be collected in which type of study to ensure that the evidence generated will meet requirements.

Broader subjects of debate are how the generation of new evidence within AEG fits with clinical research and the ethics of access to technologies for which uncertainties remain. Interest in AEG policies for coverage decisions is growing in Europe as illustrated by the work carried out by EUnetHTA WP7A (twenty countries), but also worldwide as demonstrated by the creation of the HTAi interest subgroup on conditional coverage after the 2008 HTAi meeting (30). International collaboration offers shared methodological expertise, pooled resources, possible harmonization of evidence requirements (31), less duplication, and more HTAs. It would also enable a critical mass of data to be gathered within a reasonable timeframe, especially on health technologies that concern few patients and involve long follow-ups. As a first step toward international collaboration, WP7A has developed tools for evidence sharing on promising technologies (48).

\section{CONCLUSION}

Interest in the implementation of AEG policies is growing. WP7A analyzed national experiences and has proposed a general policy framework. The main steps and key factors needed to generate relevant evidence on promising technologies were identified, and the issues they raise were discussed. International collaboration is useful, even necessary, to gather a critical mass of high-quality data quickly, while ensuring timely access to promising health technologies. The work carried out by WP7A has led to the development of tools to facilitate collaboration on evidence generation.

\section{SUPPLEMENTARY MATERIALS}

Supplementary Table 1

Supplementary Table 2

Supplementary Figure 1

www.journals.cambridge.org/thc2009003

\section{CONTACT INFORMATION}

Cédric Carbonneil, PhD (c.carbonneil@has-sante.fr), Project Manager, Fabienne Quentin, PhD (f.quentin@hassante.fr), Project Manager, Sun Hae Lee-Robin, MD, MPH (sh.leerobin@has-sante.fr), Head of Department, Medical and Surgical Procedures Assessment, French National Au- thority for Health, 2 avenue du Stade de France, Saint-Denis La Plaine CEDEX, F-93218, France

\section{REFERENCES}

1. Agence française de sécurité sanitaire des produits de santé (Afssaps). Autorisations temporaires d'utilisation. Saint Denis, France. Available at: http://www.afssaps.fr/Activites/Autorisa tions-temporaires-d-utilisation/ATU-de-cohorte/(offset)/2 (accessed December 18, 2008).

2. Agence française de sécurité sanitaire des produits de santé (Afssaps). Rapport d'activité 2007. Saint-Denis: AFSSAPS; 2007.

3. Agencia espanola de medicamentos y productos sanitarios (AEMPS). Reales Decretos 1344/2007 y 1345/2007, sobre medicamentos de uso humano y presentaciones en jornada de 29 de noviembre de 2007. Madrid, Spain. Available at: http:// www.agemed.es/actividad/legislacion/espana/realesDecretos. htm (accessed January 15, 2009).

4. Agencia Italiana del Farmaco (AIFA). Bando AIFA 2008 per la ricerca indipendente sui farmaci. Roma: AIFA; 2008.

5. Australian Government Department of Health and Ageing. Access to Unapproved Therapeutic Goods-Clinical Trials in Australia. Canberra: Australian Government Department of Health and Ageing; 2004.

6. Australian Government Department of Health and Ageing, Adverse Drug Reactions Advisory Committee (ADRAC). Joint ADRAC-Medicines Australia guidelines for the design and conduct of company-sponsored post-marketing surveillance (PMS) studies. Canberra, Australia. Available at: http://www.tga. gov.au/adr/pmsguide.htm (accessed November 12, 2008).

7. Center for Medical Technology Policy (CMTP), Schaffer DM. A multi-stakeholder approach to designing studies of emerging medical technologies. America's Health Insurance Plans (AHIP) Medical Leadership Forum, October 2007, Phoenix. [Présentation]. Baltimore. Available at: http://www.cmtpnet. org/cmtp-presentations/CMTP\% 20AHIP\% 20presentation \% 20v3\%20\%20092607\%20dms.ppt/view (accessed January 15, 2009).

8. Centers for Medicare \& Medicaid Services (CMS). InternetOnly Manuals (IOMs). Pub 100-03 Medicare National Coverage Determinations (NCD) Manual. 240-Respiratory System-240.1 Lung volume reduction surgery (reduction pneumoplasty). Baltimore. Available at: http://www.cms.hhs.gov/ manuals/downloads/ncd103c1_Part4.pdf (accessed January 15, 2009).

9. Centre fédéral d'expertise des soins de santé (KCE), Vinck I, Neyt M, et al. Procédure d'évaluation des dispositifs médicaux émergeants. Bruxelles: KCE; 2006.

10. Chalkidou K, Hoy A, Littlejohns P. Making a decision to wait for more evidence: When the National Institute for Health and Clinical Excellence recommends a technology only in the context of research. J R Soc Med. 2007;100:453-460.

11. College ter Beoordeling van Geneesmiddelen Medicines Evaluation Board (CBG-MEB). Pharmacovigilance. The Hague, The Netherlands. Available at: http://www.cbg-meb.nl/CBG/ en/human-medicines/regulatory-affairs/pharmacovigilance/ default.htm (accessed December 18, 2008). 
12. Cranovsky R, Schilling J, Faisst K, et al. Health technology assessment in Switzerland. Int J Technol Assess Health Care. 2000;16:576-590.

13. European Commission. Directive 2007/47/CE du parlement européen du conseil du 5 septembre 2007 modifiant la directive 90/385/CEE du Conseil concernant le rapprochement des législations des États membres relatives aux dispositifs médicaux implantables actifs, la directive 93/42/CEE du Conseil relative aux dispositifs médicaux et la directive 98/8/CE concernant la mise sur le marché des produits biocides. Journal Officiel de l'Union Européenne. 2007;21/09/2007.

14. European Commission-Enterprise and Industry. Post market clinical follow-up of medical devices under the medical devices directives. Medical devices: Guidance document. European Commission; 2004;2.12-2.

15. European Medicines Agency (EMEA). Guideline on risk management systems for medicinal products for human use. London: EMEA; 2005.

16. European Medicines Agency (EMEA). Guideline on the scientific application and the practical arrangements necessary to implement Commission Regulation (EC) No. 507/2006 on the conditional marketing authorization for medicinal products for human use falling within the scope of Regulation (EC) No 726/2004. London: EMEA; 2006.

17. European Medicines Agency (EMEA). The European Medicines Agency recommends suspension of the marketing authorisation of Acomplia. London, United-Kingdom. Available at: http://www.emea.europa.eu/humandocs/PDFs/EPAR/ acomplia/53777708en.pdf (accessed December 18, 2008).

18. European network for Health Technology Assessment (EUnetHTA). Timely access to promising health technologies with evidence generation: A policy framework based on national experiences. Copenhagen, Denmark. www.eunethta.net. To be published.

19. EuroScan International Network. Terminology and understanding of the activity. Birmingham, United-Kingdom. Available at: http://www.euroscan.bham.ac.uk/outputs/terminology.shtml (accessed December 18, 2008).

20. Federal Ministry of Health. Arzneimittelgesetz (AMG) Medicinal products act (the drug law) of the Federal Republic of Germany [Non-official translation]. Berlin: Federal Ministry of Health; 2006.

21. Ferreira G. Prescription-event monitoring: Developments in signal detection. Drug Saf. 2007;30:639-641.

22. Food and Drug Administration (FDA). Medical device tracking. Rockville, MD. Available at: http://www.fda.gov/ cdrh/devadvice/353.html (accessed July 5, 2006).

23. Food and Drug Administration (FDA), Booz Allen Hamilton. Postmarketing commitments study final reports. Rockville, MD: FDA; 2008.

24. Food and Drug Administration (FDA), Center for Devices and Radiological Health. Postmarket surveillance studies. Rockville, MD. Available at: http://www.fda.gov/cdrh/ devadvice/352.html (accessed July 5, 2006).

25. Global Harmonization Task Force (GHTF). Clinical evaluation. Toronto, Canada. Available at: http://www.ghtf.org/documents/ sg5/sg5_n2r8_2007final.pdf (accessed January 15, 2009).

26. Global Harmonization Task Force (GHTF). Clinical evidencekey definitions and concepts. Toronto, Canada. Available at: http://www.ghtf.org/documents/sg5/sg5_n1r8_2007final.pdf (accessed January 15, 2009).

27. Haute Autorité de Santé (HAS), Degos L. Conditional coverage of promising technologies. The French experience. HTA 2008 Conference, Montréal, Québec, Canada. [Présentation]. Saint-Denis, France. Available at: http://www.htai2008.org/ download.php?f=bc2eadd23f147d6b0e 7ff1748477 ccc0\&coun tonly $=1$ (accessed January 15, 2009).

28. Health Canada, Carleton B, Foerster V, et al. Post-marketing pharmacosurveillance in Canada. A background paper prepared for the working conference on strengthening the evaluation of real world drug safety and effectiveness. Ontario: Health Canada; 2005.

29. Health Ministry of Latvia, Health Statistics and Medical Technologies Agency (HSMTA), State Pharmaceutical Inspection, et al. Medical devices Market surveillance and Vigilance system. Riga, Latvia. Available at: http://home.arcor. de/gerda.schuett/ste-comm-07-11-05.pdf (accessed November 10, 2008).

30. Health Technology Assessment international (HTAi), Tunis $\mathrm{S}$, Chalkidou K. HTAi interest Sub-Group on conditional coverage and evidence development for promising technologies. Edmonton, Canada. Available at: http://www.htai.org/ index.php?id=89 (accessed January 15, 2009).

31. Hutton J, Trueman P, Facey K. Harmonization of evidence requirements for health technology assessment in reimbursement decision making. Int J Technol Assess Health Care. 2008;24:511-517.

32. Hutton J, Trueman P, Henshall C. Coverage with evidence development: An examination of conceptual and policy issues. Int J Technol Assess Health Care. 2007;23:425-432.

33. INFARMED-Autoridade Nacional do Medicamento e Produtos de Saúde (INFARMED I.P.). Internal regulation of the national institute of pharmacy and medicinal products. Lisboa: INFARMED I.P.; 2003.

34. Kristensen FB, Lampe K, Chase DL, et al. Practical tools and methods for health technology assessment in Europe: Structures, methodologies, and tools developed by the European network for Health Technology Assessment, EUnetHTA. Int J Technol Assess Health Care. 2009;25(Suppl 2):1-8.

35. Laupacis A, Paterson JM, Mamdani M, et al. Gaps in the evaluation and monitoring of new pharmaceuticals: Proposal for a different approach. CMAJ. 2003;169:11671170 .

36. LeLorier J. The science of health technology assessmentclinical effectiveness of therapeutic interventions. Can J Clin Pharmacol. 2001;8:21A-23A.

37. Ministerie: Indenrigs- og Sundhedsministeriet. The Danish Medicines Act, no. 1180 of 12 December 2005 with subsequent amendments. Copenhagen, Denmark. Available at: http://lmslw.lovportaler.dk/ShowDoc.aspx?docId = lov20051180uk-full (accessed December 18, 2008).

38. Ministerio de sanidad y consumo. El uso tutelado como mecanismo de actualizacion de las prestaciones: resultados de la experiencia piloto. 2007.

39. Ministerio de sanidad y consumo. Real decreto 1344/2007, de 11 de octubre, por el que se regula la farmacovigilancia de medicamentos de uso humano. Boletín Oficial del Estado. 2007;44631-44640. 
40. Ministero della Salute. Decreto ministeriale 8 maggio 2003. Uso terapeutico di medicinale sottoposto a sperimentazione clinica. Gazzetta Ufficiale. 2003;28/07/2003.

41. Ministero della Salute. Decreto Legislativo 24 aprile 2006, n. 219: "Attuazione della direttiva 2001/83/CE (e successive direttive di modifica) relativa ad un codice comunitario concernente i medicinali per uso umano, nonche' della direttiva 2003/94/CE". Gazzetta Ufficiale. 2006;21/06/2006.

42. Ministry of Health and Long-Term Care, Levin L. Coverage with evidence development workshop. Glasgow, September 19th, 2008 [Présentation]. Toronto, Canada. Available at: http://www.nhshealthquality.org/nhsqis/files/DR\%20LESLIE\% 20LEVIN.ppt (accessed January 15, 2009).

43. Ministry of Health and Long-Term Care, Levin L. Models of conditional acceptance-conditional coverage in decision making. (Workshop abstracts biotechnology and emerging technologies: The value of innovation-2 July). In: 3 rd Annual Meeting of Health Technology Assessment International (HTAi). Adelaide: HTAi; 2006.

44. National Agency for Medicines (NAM). Pharmacovigilance. Helsinki, Finland. Available at: http://www.nam.fi/ instancedata/prime_product_julkaisu/laakelaitos/embeds/Norm iuudistus_2005_M_1_2005_EN.pdf (accessed December 18, 2008).

45. National Institute for Health and Clinical Excellence (NICE), Chalkidou K. Conditional coverage: Past experience and future trends. HTAi 2008 Conference, Montréal, Québec, Canada. [Présentation]. London, United-Kingdom. Available at: http:// www.cmtpnet.org/recent-articles/topic-coverage-with-evidenc e-development/HTAi\% 202008\% 20-\% 20Chalkidou.ppt/view (accessed November 10, 2008).

46. Pharma.be. Pharmacovigilance: des médicaments sûrs et efficaces grâce à l'engagement de tous les partenaires de la santé. Factua Newsletter. 2007;1-8.

47. Programs for Assessment of Technology in Health (PATH), Goeree R, Tarride JE, et al. Using conditionally funded field evaluations (CFFE) for evidence development, uncertainty reduction and reimbursement decision making: Case studies from Ontario. HTAi 2008 Conference, Montréal, Québec, Canada. [Présentation]. Toronto, Canada. Available at: http://www. cmtpnet.org/recent-articles/topic-coverage-with-evidence-dev
elopment/HTAi\%202008\%20-\%20Goeree.pdf/view (accessed January 15, 2009).

48. Quentin F, Carbonneil C, Moty-Monnereau C, et al. Web-based toolkit to facilitate European collaboration on evidence generation on promising health technologies. Int J Technol Assess Health Care 2009;25(Suppl 2):68-74.

49. Santé Canada. L'accès aux produits thérapeutiques: Le processus de réglementation au Canada. Ontario: Santé Canada; 2006.

50. Santé Canada. Avis de conformité avec conditions (AC-C) (Produits thérapeutiques). Toronto, Canada. Available at: http://www.hc-sc.gc.ca/dhp-mps/alt_formats/hpfb-dgpsa/pdf/ prodpharma/noccfs_accfd-fra.pdf (accessed November 10, 2008).

51. Stuurgroep Weesgeneesmiddelen (WGM), van Weely S. Facilitating access to orphan treatment: The Dutch experience [Présentation]. The Hague, The Netherlands. Available at: http://www.cord.ca/index.php/site/content/download/227/948/ file/WGM\%20Canada\%20April\%2025\%202007.pdf （accessed November 10, 2008).

52. Swiss Agency for Therapeutic Products- Swissmedic. Guide to the regulation of medical devices. Bern, Switzerland. Available at: http://www.swissmedic.ch/md/files/leitf-f.html (accessed November 3, 2008).

53. The Swedish Institute for Health Economics (IHE), Persson U. Coverage with evidence development workshop experiences of Sweden. Lund, Sweden. Available at: http://www. nhshealthquality.org/nhsqis/files/DR\%20ULF\%20PERSSON. ppt (accessed January 15, 2008).

54. Tunis SR, Chalkidou K. Coverage with evidence development: A very good beginning, but much to be done. Commentary to Hutton et al. Int J Technol Assess Health Care. 2007;23:432435.

55. Tunis SR, Pearson SD. Coverage options for promising technologies: Medicare's 'coverage with evidence development'. Health Affairs (Millwood). 2006;25:1218-1230.

56. U.S. Department of Health and Human Services (HHS), Food and Drug Administration, Center for Devices and Radiological Health. Guidance for industry and FDA staff-Procedures for handling post-approval studies imposed by PMA order. Rockville, MD: FDA; 2007. 